\title{
Strictly Nonblocking Three-Stage Clos Networks With Some Rearrangeable Multicast Capability
}

\author{
Frank K. Hwang, Sheng-Chyang Liaw, and Li-Da Tong
}

\begin{abstract}
Hwang and Lin introduced a new nonblocking requirement for 2-cast traffic which imposes different requirements on different types of coexisting calls. The requirement is strictly nonblocking for point-to-point calls among the 2-cast traffic, and is rearrangeable for genuine 2-cast calls. We generalize the 2-cast calls to multicast calls and give a sufficient condition for such networks when the number of multicast calls is upper bounded.
\end{abstract}

Index Terms-Genuine multicast call, multicast call, rearrangeable, strictly nonblocking, three-stage Clos network.

\section{INTRODUCTION}

ONSIDER a three-stage $\quad$ Clos network
$C\left(n_{1}, n_{2}, r_{1}, r_{2}, m\right)$ where the input stage consists of $r_{1} n_{1} \times m$ crossbars, the middle stage $m r_{1} \times r_{2}$ crossbars, the output stage $r_{2} m \times n_{2}$ crossbars, and there exists one link between every pair of switches between two adjacent stages (see Fig. 1).

The inlets of the input switches are the inputs of the network and the outlets of the output switches are the outputs of the network. A network is called strictly nonblocking if any pair of idle input and output can be connected regardless of the existing connections of other pairs in the network (all paths must be link disjoint). A network is called rearrangeable if any set of disjoint pairs of inputs and outputs can be simultaneously connected. If the calls come sequentially, rearrangeability means we can disconnect all existing connections and reroute them together with the new call simultaneously.

Besides the point-to-point traffic as mentioned above, there is also multicast traffic where each input can request connection to many outputs. The multicast traffic is called $f$-cast if, at most, $f$ outputs can be requested in each connection. Note that the $f$-cast traffic can include point-to-point calls. We will call a multicast call with more than one output a genuine multicast call.

Paper approved by A. Pattavina, the Editor for Switching Architecture Performance of the IEEE Communications Society. Manuscript received August 28, 2001; revised March 3, 2002 and May 5, 2003. This work was supported in part by the National Science Council under Grant NSC89-2115-M-009-036, Grant NSC89-2115-M-008-31, and Grant NSC89-2115-M-029-007, and in part by the Li-Li-Tai-Yang Network Research Center of National Chiao Tung University, Hsinchu, Taiwan, R.O.C.

F. K. Hwang is with the Department of Applied Mathematics, National Chiao Tung University, Hsinchu 30050, Taiwan, R.O.C. (e-mail: fhwang@ math.nctu.edu.tw).

S.-C. Liaw is with the Department of Mathematics, National Central University, Chung Li 32054, Taiwan, R.O.C. (e-mail: scliaw@math.ncu.edu.tw).

L.-D. Tong is with the Department of Applied Mathematics, National Sun Yat-sen University, Kaohsiung 80424, Taiwan, R.O.C. (e-mail: ldtong@ math.nsysu.edu.tw).

Digital Object Identifier 10.1109/TCOMM.2003.819215

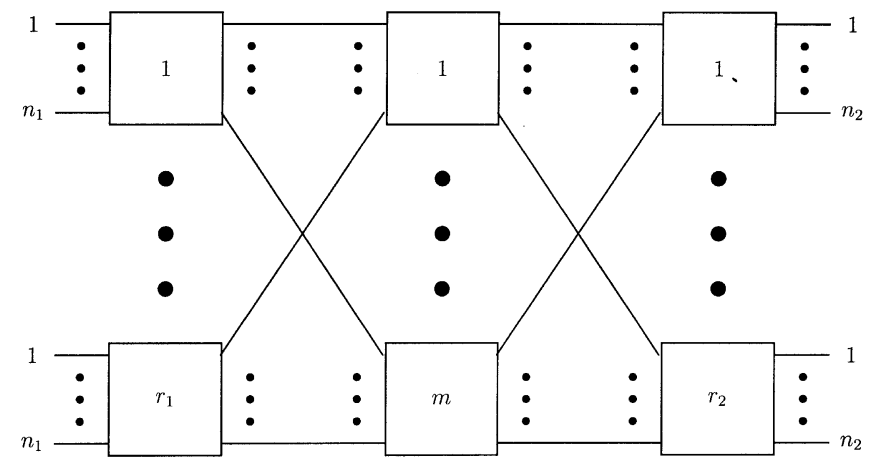

Fig. 1. $C\left(n_{1}, n_{2}, r_{1}, r_{2}, m\right)$.

Hwang and Lin [6] consider a three-stage Clos network in the situation where the predominant use is point-to-point traffic, but occasionally the network is requested to carry some multicast traffic. In particular, they conjectured the following.

Conjecture 1: $C(n, r, 2 n)=C(n, n, r, r, 2 n)$ is rearrangeable for 2-cast calls and strictly nonblocking for the point-topoint calls among them.

It is well known [1] that $C\left(n_{1}, n_{2}, r_{1}, r_{2}, m\right)$ is strictly nonblocking if $m \geq n_{1}+n_{2}-1$. It is also well known [7] that $C\left(n_{1}, n_{2}, r_{1}, r_{2}, m\right)$ is $f$-cast rearrangeable if $m \geq f n_{1}$. Therefore, $C(n, r, 2 n)$ is indeed strictly nonblocking if all traffic is point-to-point, and rearrangeable if all traffic is 2-cast. However, this does not imply that when the traffic contains both point-to-point calls and genuine 2-cast calls, $C(n, r, 2 n)$ can still be strictly nonblocking for each point-to-point call and rearrangeable for each genuine 2-cast call. This is because the routing for the "strictly nonblocking" part assumes each connection, point-to-point or 2-cast, to use only one outlet of the involved input switch, while the routing for the "rearrangeable" part assumes each genuine 2-cast call to use two such outlets. Thus, the two routings conflict with each other in how to route a 2-cast call. To preserve the strict nonblockingness for point-topoint calls, we must find a new routing for genuine 2-cast calls in which the input switches do not fan out a connection (with the fringe benefit that the input switches do not need fan-out capability).

In this letter, we prove a result related to Conjecture 1. It is a special case of Conjecture 1 in the sense that the number of multicast calls is restricted. Note that this special case fits the intended application when multicast calls are occasional. Our result is more general than Conjecture 1 in the sense that we deal with the more general $C\left(n_{1}, n_{2}, r_{1}, r_{2}, m\right)$, we require a smaller $m$, and we handle multicast, not just 2-cast, calls. 


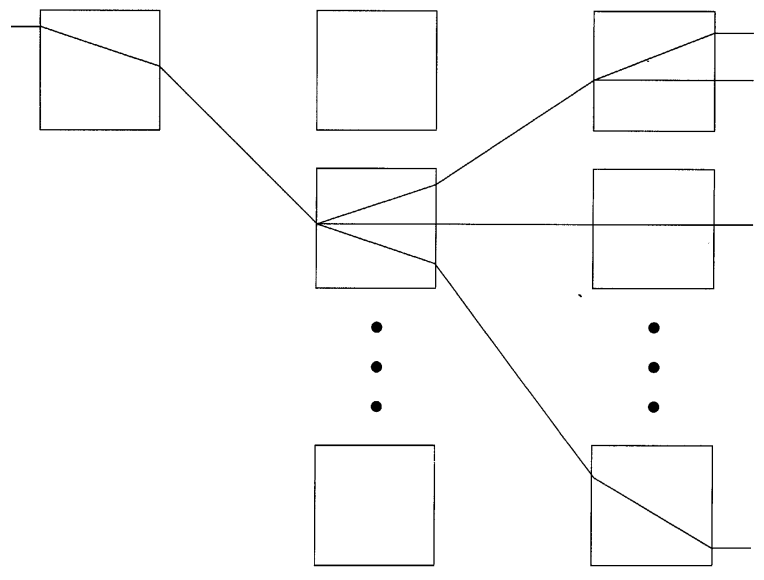

Fig. 2. Connection of a multicast call.

\section{THE MAIN Result}

Let $M$ denote the number of multicast calls in the network, including those already connected and the newly arrived.

Theorem 1: $C\left(n_{1}, n_{2}, r_{1}, r_{2}, m\right)$ with $M \leq m$ is rearrangeable for multicast calls and strictly nonblocking for the point-topoint calls among them if and only if $m \geq \min \left\{n_{1}+n_{2}-\right.$ $\left.1, n_{1} r_{1}, n_{2} r_{2}\right\}$.

Proof: "if." Since $n_{1} r_{1}$ and $n_{2} r_{2}$ are trivially sufficient for nonblocking, we show that $n_{1}+n_{2}-1$ is also enough. We first prove the "rearrangeability" part. When a multicast call arrives, we disconnect all existing calls, and then route all multicast calls, at most, $m$ of them, each to a distinct middle switch. Using the fan-out property of a crossbar, the connection splits $f$ ways in that middle switch to go to the $f$ destination output switches. An output switch containing $g$ destinations will have $g$ splits within that switch (see Fig. 2). Note that each multicast call consumes one outlink of the involved input switch, and one inlink of each involved output switch. Next we connect the point-to-point call. Suppose we are connecting a call from input switch $I$ to output switch $O$. Due to the way we route the multicast calls, regardless of whether the other $n_{1}-1$ inlets of $I$ are connected, and whether these connections are point-to-point or multicast, at most, $n_{1}-1$ outlinks of $I$ are consumed. Similarly, at most, $n_{2}-1$ inlinks of $O$ are consumed. Since

$$
\left(n_{1}-1\right)+\left(n_{2}-1\right)<n_{1}+n_{2}-1
$$

there must exist a middle switch with open links to both $I$ and $O$, and can be used to carry the $(I, O)$ call. Next we prove the "strict nonblockingness" part. Note that the above argument to route a point-to-point call is valid regardless of how previous point-to-point calls are connected. Therefore, that argument can also be used to prove that the point-to-point calls are strictly nonblocking.

"only if." Since $m \geq \min \left\{n_{1}+n_{2}-1, n_{1} r_{1}, n_{2} r_{2}\right\}$ is necessary for strict nonblockingness for the special case when all calls are point-to-point, it is also necessary for the general multicast traffic.

The $M \leq m$ restriction applies only when $\min \left\{n_{1}+n_{2}-\right.$ $\left.1, n_{1} r_{1}, n_{2} r_{2}\right\}=n_{1}+n_{2}-1$. We next show that this restriction can be dropped if $n_{1}$ is much larger than $n_{2}$.
Corollary 1: $C\left(n_{1}, n_{2}, r_{1}, r_{2}, n_{1}+n_{2}-1\right)$ is rearrangeable for multicast calls and strictly nonblocking for the point-to-point calls among them, if $n_{1} \geq k n_{2}$ for some integer $k$, and $r_{2} \leq$ $k+3$, except for $n_{1}=n_{2}$.

Proof:

$$
\begin{aligned}
M & \leq\left\lfloor\frac{r_{2} n_{2}}{2}\right\rfloor \leq\left\lfloor\frac{(k+3) n_{2}}{2}\right\rfloor \\
& \leq\left\lfloor\frac{n_{1}+3 n_{2}}{2}\right\rfloor \leq n_{1}+n_{2}-1 .
\end{aligned}
$$

Corollary 2: $C(n, r, 2 n)$ is rearrangeable for multicast calls and strictly nonblocking for the point-to-point calls among them if $r \leq 4$.

Proof:

$$
M \leq \frac{r n}{2} \leq 2 n
$$

From the proof of Theorem 1, we know that if there is a way to route all multicast calls by using, at most, $n_{1}+n_{2}-1$ middle switches, then we can properly route the remaining point-topoint calls with strictly nonblocking properties. In [5], Hwang and Liaw have shown that $C\left(n_{1}, n_{2}, r_{1}, r_{2}, n_{1}+f\left(n_{2}-1\right)\right)$ is strictly nonblocking for $f$-cast. From their argument, we know that $k+f\left(n_{2}-1\right)$ middle switches are enough to route $f$-cast calls, with each input switch having, at most, $k$ multicast requests. These results suggest the following theorem to replace the bound on the total number of multicast calls by the bound on multicast calls in each input switch.

Theorem 2: $C\left(n_{1}, n_{2}, r_{1}, r_{2}, \max \left\{k+f\left(n_{2}-1\right), n_{1}+n_{2}-\right.\right.$ $1\})$ is rearrangeable for multicast calls and strictly nonblocking for the point-to-point calls among them if each call can have, at most, $f$ outputs, and each input switch has, at most, $k$ genuine multicast requests.

We can take advantage of Theorem 2 to design a three-stage Clos network with $n_{1}$ much larger than $n_{2}$ so that more genuine multicast calls can be accomodated. For example, for $f=2$, then by Theorem 2, $n_{1}+n_{2}-1$ middle switches are enough for the mixed traffic with each input switch having, at most, $n_{1}-$ $n_{2}+12$-cast calls. So, the total number of 2-cast calls allowed can be much larger than $n_{1}+n_{2}-1$, as given by Theorem 1 .

\section{SOME Related Literature}

Some special cases of Conjecture 1 have been proved. For example, Du and Ngo [3] proved for $n_{2}=2$ (except $n_{1}=3$ ) and $n_{2}=3$. They also formulated a graph-theoretic conjecture based on Conjecture 1.

Conjecture 2: Consider a graph $H$ with maximum degree $n_{2}$. Let $L(H)$ be the line graph of $H$. Divide all vertices of $L(H)$ into disjoint groups of size, at most, $n_{1}$. Connect all vertices in each group into a clique. If $n_{2} \leq n_{1}$, then the resulting graph is $\left(n_{2}+n_{1}\right)$ colorable.

Here, $H$ is the graph with output switches as vertices and a link $(u, v)$ represents a 2-cast call involving output switches $u$ and $v$.

Conjecture 2 extends another conjecture by Du et al. [2] which was eventually proved by Fleischner and Stiebitz [4].

Conjecture 3: A graph consisting of a $3 n$ cycle and $n$ disjoint triangles is $n$ colorable. 


\section{REFERENCES}

[1] C. Clos, "A study of nonblocking switching networks," Bell Syst. Tech. J., vol. 32, pp. 406-424, 1953.

[2] D. Z. Du, D. F. Hsu, and F. K. Hwang, "The Hamiltonian property of consecutive-d digraphs," Math. Comput. Modeling, vol. 17, pp. 61-63, 1993.

[3] D. Z. Du and H. Q. Ngo, "An extension of DHH-erdös conjecture on cycle-plus-triangle graphs," Taiwan J. Math., to be published.
[4] H. Fleischner and M. Stiebitz, "A solution to a coloring problem of P. Erdös," Disc. Math., vol. 101, pp. 39-48, 1992.

[5] F. K. Hwang and S. C. Liaw, "On nonblocking multicast 3-stage Clos networks," IEEE/ACM Trans. Networking, vol. 8, pp. 535-539, 2000.

[6] F. K. Hwang and C. H. Lin, "Broadcasting in a three-stage point-to-point nonblocking network," Int. J. Rel. Qual. Safety Eng., vol. 2, pp. 299-307, 1995.

[7] G. M. Masson and B. W. Jordan, "Generalized multi-stage connection networks," Networks, vol. 2, pp. 191-209, 1972. 